\title{
Ventilation heterogeneity in children with well controlled asthma with normal spirometry indicates residual airways disease
}

\author{
K A Macleod, ${ }^{1,3}$ A R Horsley, ${ }^{2,3} \mathrm{~N}$ J Bell, ${ }^{2,3}$ A P Greening, ${ }^{2,3} \mathrm{~J} \mathrm{~A} \mathrm{Innes,}^{2,3}$ S Cunningham ${ }^{1}$
}

${ }^{1}$ Department of Respiratory, Sleep and General Medicine, Royal Hospital for Sick Children, Edinburgh, UK: ${ }^{2}$ Respiratory Unit, Western General Hospital, Edinburgh, UK; ${ }^{3}$ Molecular Medicine Centre, University of Edinburgh, Edinburgh, UK

Correspondence to: Dr K A Macleod, Department of Respiratory, Sleep and General Medicine, Royal Hospital for Sick Children, Sciennes Road, Edinburgh EH9 1LF, UK: kenny.macleod@ed.ac.uk

Received 19 December 2007 Accepted 29 June 2008 Published Online First

4 August 2008
ABSTRAC

Background: In adults with asthma, ventilation heterogeneity, independent of inflammation, has been hypothesised to be associated with airway remodelling. Bronchial biopsy in preschool children with wheeze demonstrates early structural changes. Ventilation heterogeneity is sensitive to airway disease in other paediatric respiratory conditions such as cystic fibrosis, so may be sensitive to early airway disease in asthma. An observational study was performed in which it was hypothesised that ventilation heterogeneity (lung clearance index (LCI) and phase III slope indices $\left(S_{\text {cond }}\right.$ and $S_{\text {acin }}$ )/ were more sensitive than conventional measurements (forced expiratory volume in $1 \mathrm{~s}\left(\mathrm{FEV}_{1}\right)$ and exhaled nitric oxide (Feno)) for detecting residual airways disease in children with well controlled asthma.

Methods: In 31 children with asthma of mean age 10.6 years (range $5-15$ ), $\mathrm{FEV}_{1}, \mathrm{LCl}, \mathrm{S}_{\text {cond }}$ and $\mathrm{S}_{\text {acin }}$ were measured at two separate visits, before and after blinded salbutamol or placebo, with Feno measured once. 29 healthy volunteers of mean age 11.2 years (range 5-16) completed measurements at one visit only.

Results: Baseline mean (SD) LCI was significantly higher in children with asthma than in controls (6.69 (0.91) vs $6.24(0.47), p=0.02)$. There were no significant differences in $\mathrm{FEV}_{1}$ or median Feno. Following salbutamol there was a small significant change in mean (SD) FEV (from $-1.26(1.25)$ to $-0.93(0.23), p=0.03$ ) but not in $\mathrm{LCl}, \mathrm{S}_{\text {cond }}$ or $\mathrm{S}_{\text {acin. }}$ Importantly, $\mathrm{LCl}$ remained significantly higher after bronchodilator in children with asthma than in controls (6.64 (0.69), $p=0.01$ ).

Conclusion: This study identifies the presence of residual ventilation heterogeneity in children with well controlled asthma and normal $\mathrm{FEV}_{1}$. The role of $\mathrm{LCl}$ in measuring early airway disease in children with asthma requires further exploration, possibly as a surrogate of structural remodelling.

Asthma has a variable course in childhood with evidence of distinct phenotypes. ${ }^{1}$ The characteristic features of asthma (airway inflammation and hyper-responsiveness) are apparently controllable with treatment, leading to minimal symptoms in most cases. It is currently unclear how early childhood events or genetic factors contribute to disease progression, as even individuals with well controlled disease may continue to have ongoing histological evidence of airway obstruction and inflammation. ${ }^{2}$ Structural changes to the airway wall and vasculature (airway remodelling) may explain why some patients with good symptom control in adolescence appear to have accelerated decline in lung function in later adult life. ${ }^{4}$ These airway structural changes have recently been noted to be absent in infants ${ }^{5}$ but have developed as early as the immediate preschool years. ${ }^{6}$ If this process of remodelling is to be influenced by treatments, then non-invasive markers of airway remodelling require to be identified as airway biopsy is an invasive and poorly repeatable measure.

Disease severity in asthma is conventionally assessed using spirometry (forced expiratory volume in $1 \mathrm{~s}\left(\mathrm{FEV}_{1}\right)$ ) and symptoms. Current opinions suggest that spirometry is able to detect airway obstruction and reversibility in the medium to large airways but may be insensitive to subtle changes in the distal airways. ${ }^{78}$ Young children also have difficulty in performing spirometry as it requires cooperation and learned techniques. In children with cystic fibrosis, spirometry is poorly sensitive to early airway disease. ${ }^{9}$ Sensitive and repeatable measures of pulmonary function are now being developed to monitor and track small airways disease from infancy to adulthood. ${ }^{10}{ }^{11}$

The lung clearance index (LCI) is a simple measure of ventilation heterogeneity derived from multiple breath inert gas washout measurements. This technique is well suited to all age groups, requiring simple tidal breathing. LCI has a narrow range of normal that does not seem to change throughout life, ${ }^{12}$ and in children it has been shown to be more sensitive than $\mathrm{FEV}_{1}$ at detecting early airways disease, particularly in cystic fibrosis. ${ }^{14} 15$ Two additional indices of ventilation heterogeneity can be derived from the analysis of progressive changes in the phase III (alveolar) slope with each breath of the washout. These indices are considered to correspond to abnormalities in the conductive airways ( $\left.S_{\text {cond }}\right)$ and acinar airways $\left(S_{\text {acin }}\right)$, respectively. $S_{\text {cond }}$ and $S_{\text {acin }}$ have been used to demonstrate changes in ventilation heterogeneity in children with asthma and cystic fibrosis. ${ }^{13} 16-20$

A study in adults with asthma demonstrated residual ventilation heterogeneity, independent of inflammation. ${ }^{18}$ This is postulated as representing airway remodelling. Only one study has previously assessed ventilation heterogeneity in children with asthma. ${ }^{16}$ These children had a poor baseline $\mathrm{FEV}_{1}$ and hence would not be considered well controlled by current standards. LCI and $\mathrm{FEV}_{1}$ improved after bronchodilator treatment but remained significantly different from controls.

We conducted a randomised placebo controlled observational study to assess residual ventilation heterogeneity (LCI) in children with well controlled asthma following bronchodilator compared at baseline with healthy controls. Our aim was to 
assess whether residual ventilation heterogeneity, possibly representing evidence of airway remodelling, is present in children with well controlled asthma.

\section{METHODS}

\section{Patients}

Children with asthma aged 5-16 years were recruited from those attending the Royal Hospital for Sick Children specialist asthma clinic. Inclusion criteria were known asthma diagnosed by a consultant respiratory paediatrician, no previous history of severe asthma requiring admission to intensive care and no increase in symptoms or any exacerbation requiring increased use of inhaled bronchodilator or systemic steroid treatment in the 4 weeks prior to testing. Age-matched healthy volunteers were recruited from patients attending for follow-up of stable upper limb fractures as well as children of hospital staff. Healthy volunteers had no history of recurrent wheeze, pertussis or tuberculosis; no previous diagnosis of asthma or previous use of asthma medication; no prematurity $(<34$ completed weeks gestation); no neuromuscular weakness or bone disease likely to affect respiration; and no congenital cardiac disease requiring medication.

\section{Study design}

Patients with asthma attended on two separate occasions 1 week apart. All patients took normal preventer medication on the day of testing but were asked to withhold short-acting $\beta_{2}$ agonists for $6 \mathrm{~h}$ prior to testing. At each visit multiple breath washout (MBW) and standard spirometry were completed before and 20 min after inhaled salbutamol (200 $\mu$ g metered dose inhaler (MDI), Allen and Hanburys, GlaxoSmithKline (GSK), UK) or placebo (propellant only MDI, Allen and Hanburys, GSK, UK). Both salbutamol and placebo were delivered via standard spacer (Aerochamber plus, GSK, UK). In addition, exhaled nitric oxide (FeNO) was measured at each visit after the first spirometry assessment. Patients were administered salbutamol or placebo in a randomised double-blind manner with the alternative intervention given at the second visit to ensure any variability in tests was valid. Salbutamol and placebo MDIs were masked by an independent person. The order of intervention was determined by sealed sequentially numbered opaque envelopes, also prepared independently of the study team.

Healthy volunteer subjects attended on one occasion to complete MBW, spirometry and FeNO with no intervention.

Approval for the study was given by the Lothian Research Ethics Committee.

\section{Multiple breath washout (MBW)}

MBW was measured using the modified Innocor flow meter and side stream gas analyser (Innovision, Denmark) with the addition of a screen pneumotach (Hans Rudolph, USA), as previously validated for use in adults and children as young as 5 years of age. ${ }^{12}$ Briefly, subjects were suitably distracted and seated with a nose clip attached as they breathed $0.2 \%$ sulphur hexafluoride $\left(\mathrm{SF}_{6}\right)$ in medical air, supplied from a compressed gas cylinder (BOC, Hertford, UK) via a mouthpiece and flow past (bias flow) system until expired and inspired concentrations had equilibrated. The gas supply was then detached and patients breathed room air until the measured end-tidal $\mathrm{SF}_{6}$ concentration was $1 / 40$ th of the starting concentration $(0.005 \%)$. Tidal breathing was used throughout the test, each taking 5-10 min to complete. Washouts were repeated three times at each stage to obtain average values. ${ }^{17}$

Functional residual capacity (FRC) is derived from the total volume of expired tracer gas and starting gas concentration. ${ }^{21}$ LCI is defined as the total cumulative expired volume (CEV) required to wash the tracer gas out to $1 / 40$ th of the starting value, divided by the measured FRC (CEV/FRC). In other words, LCI is the number of starting lung volume turnovers required to wash out the $\mathrm{SF}_{6}$ to the predefined point and is raised in lungs with increased ventilation heterogeneity.

$\mathrm{S}_{\text {cond }}$ and $\mathrm{S}_{\text {acin }}$ were derived from plots of the phase III slope against lung turnover normalised for mean expired $\mathrm{SF}_{6}$ concentration over that portion of the corresponding breath. A full description of this technique is given by Verbanck et al. ${ }^{22}$ Tidal volume was not restricted in our patients. In order to allow comparison of the data with published work in adults who were normally limited to breaths of 1 litre, individual normalised phase III slope values were corrected by multiplying by the breath volume before calculating $S_{\text {cond }}$ and $S_{\text {acin. }} \cdot{ }^{13}{ }^{17}$ Following this protocol, negative $S_{\text {cond }}$ values are seen in both healthy volunteers and those with very mild disease. While this is biologically implausible in theory, negative values are seen in practice owing to variation in tidal volume throughout the washout and lack of progression of the normalised phase III slope. This is consistent with other studies in paediatric populations using a similar washout protocol. ${ }^{13}$

LCI, FRC, $\mathrm{S}_{\text {cond }}$ and $\mathrm{S}_{\text {acin }}$ are all quoted as the mean of at least two reproducible measurements out of three washouts. As a quality control measure, washouts were excluded if the measured FRC differed by more than $10 \%$ from both other repeats. ${ }^{17}$

\section{Spirometry}

Spirometry (Easyone; nnd, Sweden or Micromedical, UK) was performed according to ARTP guidelines. ${ }^{23}$ Measurements were performed standing up, without a noseclip. Three reproducible measures (defined according to ERS/ATS standards) ${ }^{24}$ were required for a satisfactory result, with the best of three recorded. Standard deviation scores (z-scores) were calculated from the reference population residual standard deviation. ${ }^{25}$

\section{Exhaled nitric oxide}

The fraction of expired nitric oxide (FenO; parts per billion $(\mathrm{ppb})$ ) was measured using the online NioxMino analyser (Aerocrine, Sweden). This device requires the child to breathe in to full vital capacity through a mouthpiece, then to breathe out steadily against resistance. A single acceptable measurement was performed with a flow rate of $5 \pm 5 \mathrm{ml} / \mathrm{s}$ and exhalation time $6 \mathrm{~s}$, according to ATS standards. ${ }^{26}$ An upper limit of $20 \mathrm{ppb}$ is considered abnormal.

\section{Statistical analysis}

Data were analysed using Minitab Version 14 statistical software (Minitab, USA). Data are presented as mean with standard deviation (SD) unless otherwise stated. The Student $t$ test for parametric data and Mann-Whitney test for nonparametric data were used for comparison of groups and effect of treatment. Correlations between two measures were assessed using Pearson correlation coefficient. Non-parametric paired comparisons were analysed using the two-sample Wilcoxon signed rank test. $p$ Values $<0.05$ were considered significant. 
Table 1 Characteristics and baseline measurements of study subjects at first visit

\begin{tabular}{|c|c|c|c|}
\hline & Healthy controls & $\begin{array}{l}\text { Asthmatic patients at } \\
\text { first visit baseline }\end{array}$ & p Value \\
\hline Number & $29\left(28\right.$ for $S_{\text {cond }}$ and $\left.S_{\text {acin }}\right)$ & 31 & \\
\hline Mean (range) age (years) & $11.1(5-16)$ & $10.6(5-15)$ & 0.54 \\
\hline $\operatorname{Sex}(M / F)$ & $18 / 11$ & $17 / 14$ & \\
\hline \multicolumn{4}{|l|}{ Medication at time of testing } \\
\hline ICS & - & $6 \%$ & \\
\hline $\mathrm{ICS}+\mathrm{LABA}$ & - & $52 \%$ & \\
\hline ICS+montelukast & - & $42 \%$ & \\
\hline \multicolumn{4}{|l|}{ Atopy } \\
\hline Positive skin prick testing to aeroallergen & - & $58 \%$ & \\
\hline Eczema & - & $16 \%$ & \\
\hline Hay fever & - & $71 \%$ & \\
\hline Mean (range) height $(\mathrm{cm})$ & $148(118-180)$ & $144(110-186)$ & 0.38 \\
\hline Median (range) Feno (ppb) & $18(3-50)$ & $14(5-300)$ & $0.63^{*}$ \\
\hline Mean (SD) FEV ${ }_{1}$ z-score & $-0.69(0.88)$ & $-1.09(1.28)$ & 0.16 \\
\hline Range & $-2.64-1.28$ & $-4.00-1.82$ & \\
\hline Mean (SD) LCI (CEV/FRC) & $6.24(0.47)$ & $6.69(0.91)$ & 0.02 \\
\hline Range & $5.14-7.05$ & $5.49-9.46$ & \\
\hline Mean (SD) $S_{\text {cond, }}$ Vt corrected & $0.017(0.02)$ & $0.026(0.02)$ & 0.06 \\
\hline Range & $-0.03-0.06$ & $-0.01-0.09$ & \\
\hline Mean (SD) $\mathrm{S}_{\text {acin, }}$ Vt corrected & $0.12(0.06)$ & $0.14(0.02)$ & 0.23 \\
\hline Range & $0.02-0.29$ & $0.05-0.40$ & \\
\hline Mean (SD) Vt (I) & $0.55(0.24)$ & $0.45(0.17)$ & 0.07 \\
\hline Range & $0.19-0.99$ & $0.23-0.80$ & \\
\hline Mean (SD) FRC (I) & $2.14(1.02)$ & $1.91(0.78)$ & 0.31 \\
\hline Range & $0.80-4.51$ & $0.85-3.82$ & \\
\hline Mean (SD) Vd/Vt ratio & $0.27(0.06)$ & $0.27(0.05)$ & 0.89 \\
\hline Range & $0.13-0.37$ & $0.17-0.36$ & \\
\hline
\end{tabular}

Comparison of groups at baseline.

CEV, cumulative expired volume; Feno, fraction of expired nitric oxide; $\mathrm{FEV}_{1}$, forced expiratory volume in $1 \mathrm{~s}$; $F R C$, functional residual capacity; ICS, inhaled corticosteroid; LABA, long-acting $\beta_{2}$ agonist; LCI, lung clearance index; $S_{\text {acin, }} \mathrm{S}_{\text {cond, }}$ phase III (alveolar) slope of the acinar and conductive airways, respectively; $\mathrm{Vd}$, dead space; $\mathrm{Vt}$, tidal volume.

*Mann-Whitney non-parametric test.

\section{RESULTS}

\section{Subjects}

Thirty-one patients with asthma of mean age 10.6 years (range $5-15$ ) and 29 healthy volunteers of mean age 11.1 years (range 5-16) were recruited to the study. Five patients with asthma withdrew from the study after one visit (two suffered exacerbations and three were unable to find time to return). All participants completed the measurements without difficulty.

All patients with asthma were taking regular inhaled corticosteroids at the time of testing. $6 \%$ of patients were at step 2 of the BTS guidelines for paediatric asthma management $^{27}$ (regular inhaled corticosteroids 200-400 $\mu \mathrm{g} /$ day), 68\% were on step 3 (regular inhaled corticosteroids $200-400 \mu \mathrm{g} /$ day with long acting $\beta_{2}$ agonist (LABA) or montelukast) and $26 \%$ were on step 4 (regular inhaled corticosteroids $>400 \mu \mathrm{g} /$ day \pm LABA or montelukast); $58 \%$ of patients had evidence of atopy (positive skin prick testing to aeroallergen with a history of hay fever and/or eczema).

\section{Baseline data}

Patient demographic characteristics and baseline measurements from the first attendance in the study are shown in table 1. Groups were matched for age, sex and height. The mean (SD) baseline $\mathrm{FEV}_{1}$ z-score at the first visit was not statistically lower in the patients with asthma than in the controls $(-1.09(1.28)$ vs $-0.70(0.88), p=0.16)$. Both groups were within the normal range $( \pm 1.96 \mathrm{SD})$. There was also no difference in median

Table 2 Measurements before and 20 min after salbutamol in children with asthma compared with healthy controls

\begin{tabular}{|c|c|c|c|c|}
\hline & \multirow[b]{2}{*}{ Healthy controls } & \multicolumn{3}{|c|}{ Asthmatic children } \\
\hline & & Pre-salbutamol & Post-salbutamol & $\begin{array}{l}\text { p Value (post- } \\
\text { salbutamol vs } \\
\text { pre-salbutamol) }\end{array}$ \\
\hline Mean (SD) FEV ${ }_{1}$ z-score & $-0.69(0.88)$ & $-1.26(1.25)$ & $-0.93(0.23)$ & 0.03 \\
\hline Mean (SD) LCI & $6.24(0.47)$ & $6.82(1.04)$ & $6.64(0.69)^{*}$ & 0.12 \\
\hline Mean (SD) $\mathrm{S}_{\text {cond }}$ & $0.017(0.02)$ & $0.027(0.02)$ & $0.020(0.04)$ & 0.19 \\
\hline Mean (SD) $S_{\text {acin }}$ & $0.12(0.06)$ & $0.12(0.06)$ & $0.14(0.10)$ & 0.45 \\
\hline
\end{tabular}

$\mathrm{FEV}_{1}$, forced expiratory volume in $1 \mathrm{~s}$; LCl, lung clearance index; $\mathrm{S}_{\text {acin, }} \mathrm{S}_{\text {cond, }}$ phase III (alveolar) slope of the acinar and conductive airways, respectively.

${ }^{*}$ Significant difference in post-salbutamol value compared with healthy controls $(p=0.01)$. 
Table 3 Measurements before and 20 min after placebo in children with asthma

\begin{tabular}{lccc}
\hline & \multicolumn{2}{c}{ Asthmatic children } & \\
\cline { 2 - 4 } & Pre-placebo & Post-placebo & $\begin{array}{l}\text { p Value (post-placebo } \\
\text { vs pre-placebo) }\end{array}$ \\
\hline Mean (SD) FEV 1 z-score & $-1.036(1.14)$ & $-0.97(1.17)$ & 0.45 \\
Mean (SD) LCl & $6.69(1.01)$ & $6.71(1.23)$ & 0.83 \\
Mean (SD) S & $0.033(0.03)$ & $0.033(0.02)$ & 0.90 \\
Mean (SD) S & $0.14(0.09)$ & $0.16(0.12)$ & 0.46 \\
\hline
\end{tabular}

FEV $_{1}$, forced expiratory volume in $1 \mathrm{~s}$; $\mathrm{LCl}$, lung clearance index; $\mathrm{S}_{\text {acin, }} \mathrm{S}_{\text {cond, }}$ phase III (alveolar) slope of the acinar and conductive airways, respectively.

(range) FeNO (ppb) between children with asthma and controls (14 (5-300) vs 18 (3-50), $p=0.63)$.

Mean (SD) baseline LCI was higher in the asthmatic group than in the controls at visit 1 (6.69 (0.91) vs 6.24 (0.47), $\mathrm{p}=0.02)$, indicating greater overall ventilation heterogeneity. There was a trend towards higher $S_{\text {cond }}$ in the asthma group, although this was not significant $(0.026(0.02)$ vs $0.017(0.02)$, $p=0.06)$. There was no difference in $S_{\text {acin }}$ between the groups (0.14 (0.02) vs $0.12(0.06), p=0.23)$. There was no statistical difference in mean tidal volume, FRC or dead space (Fowler method) to tidal volume ratio $(\mathrm{Vd} / \mathrm{Vt})$.

There was no correlation between age and either LCI, $\mathrm{S}_{\text {cond }}$ or $S_{\text {acin }}$ in healthy volunteers or children with asthma. Increased LCI did not correlate with increased steroid dose or BTS step of asthma treatment.

At visit 1, prior to intervention, there was no correlation between LCI and $\mathrm{FEV}_{1}$ z-score in patients with asthma $\left(\mathrm{r}^{2}=0.11, \mathrm{p}=0.08\right)$. There was a significant correlation between LCI and $S_{\text {cond }}\left(r^{2}=0.41, p<0.001\right)$ but not between LCI and $S_{\text {acin }}\left(r^{2}=0.11, p=0.07\right)$. This relationship between LCI and $\mathrm{S}_{\text {cond }}$ was maintained at each time point on both visits. There were no significant correlations between FenO and any of the measured physiological variables.

To assess repeatability of measurements in patients with asthma, within-subject coefficient of variation (CV, withinsubject standard deviation divided by population mean) was calculated for $\mathrm{FEV}_{1}$ and LCI before and after placebo (4.17\% and $5.39 \%$, respectively).

\section{Salbutamol intervention}

Following salbutamol administration there was a significant increase $(6 \%)$ in mean (SD) $\mathrm{FEV}_{1} \mathrm{z}$-score (from -1.26 (1.25) to -0.93 (0.23), $p=0.03)$, indicating additional reversibility despite optimal asthma control. No change was seen after placebo $(-1.04$ (1.14) to $-0.97(1.17), p=0.5)$. There was no significant change in mean LCI, $S_{\text {acin }}$ or $S_{\text {cond }}$ following either salbutamol or placebo (tables 2 and 3 ).

The mean (SD) post-salbutamol $\mathrm{FEV}_{1}$ z-score did not differ significantly from healthy controls $(-0.93(0.23)$ vs $-0.69(0.88)$ $\mathrm{p}=0.38)$, but the mean (SD) post-salbutamol LCI remained significantly higher than in controls (6.64 (0.69) vs 6.24 (0.47), $\mathrm{p}=0.01$ ), indicating residual overall ventilation heterogeneity despite improvement in $\mathrm{FEV}_{1}$. Compared with controls, there was no difference in post-salbutamol $S_{\text {cond }}(p=0.54)$ or $S_{\text {acin }}$ $(p=0.33$; table 2$)$.

\section{DISCUSSION}

In this study, children with well controlled asthma and normal $\mathrm{FEV}_{1}$ had significantly higher LCI than controls, indicating abnormal gas mixing. As $S_{\text {cond }}$ correlated with LCI at all time points, this abnormality appeared to be located in the conducting airways $\left(\mathrm{S}_{\text {cond }}\right)$ as opposed to the acini $\left(\mathrm{S}_{\mathrm{acin}}\right)$, although the trend towards a difference in $S_{\text {cond }}$ between the groups did not reach statistical significance $(p=0.06)$.

In patients with ongoing inhaled corticosteroid therapy and a high level of asthma control, salbutamol administration only minimally improved $\mathrm{FEV}_{1}$, which is to be expected in a population with well controlled disease. Despite this, postsalbutamol mean LCI remained significantly raised, indicating the presence of residual small airways disease not detected by $\mathrm{FEV}_{1}$.

This study shows, for the first time, evidence of ventilation heterogeneity in children with well controlled asthma and normal FEV 1 . This residual disease did not appear to reflect persisting inflammation or bronchospasm as FeNO was normal and the heterogeneity persisted after salbutamol administration. It is possible therefore that LCI may reflect structural airway changes consistent with previous observations made by biopsy in children with asthma. ${ }^{5}$ Further studies would be required to directly link these two observations.

This observational study is limited in that it is unable to directly link ventilation heterogeneity with independent evidence of structural airway changes. Given the difficulty in performing these invasive measurements, we can only hypothesise as to the cause of this residual ventilation heterogeneity. Measurement of airway hyper-responsiveness (AHR) may also have provided further evidence of airway remodelling to support our hypothesis. If we had recruited preschool children we may have been able to determine the influence of age and length of time with wheeze on ventilation heterogeneity.

In adults with asthma, ventilation heterogeneity has been shown to correlate with AHR, independent of airway inflammation measured by exhaled nitric oxide. ${ }^{18}$ In a similar study in adults with mild asthma reported by Verbanck et al, $S_{\text {cond }}$ and $S_{\text {acin }}$ were measured before and after salbutamol. As in our study, there was evidence of ventilation heterogeneity in the conducting airways but not in the acini. ${ }^{19}$ The persistently raised $S_{\text {cond }}$ following salbutamol reinforces the suggestion of non-bronchodilator responsive disease in the small conducting airways of patients with well controlled asthma. The increase in $\mathrm{S}_{\text {cond }}$ was greater in both of these studies than in our study. This may be because the asthma was less well controlled than in our patients or due to longer duration of asthma leading to greater progression of airway structural changes. Gustafsson demonstrated increased LCI and $S_{\text {cond }}$ in a Swedish population of children with moderately severe asthma, with a greater improvement in both of these variables than we have observed. ${ }^{16}$ Compared with our study, however, these patients had a lower baseline $\mathrm{FEV}_{1}$ (77\% predicted) and a higher mean LCI $(>8)$.

Inflammation is an important component of asthma. When exhaled nitric oxide or sputum eosinophil count are used to titrate inhaled steroid doses in adults with asthma, better control can be achieved with fewer exacerbations and an overall 
lower steroid dose than using symptoms alone. ${ }^{28} 29$ Similarly, in children aged 6-18 years, the used of FenO as the basis for increasing or decreasing the dose of inhaled steroid resulted in improved AHR and fewer exacerbations than those treated by symptoms alone. ${ }^{30}$ While inflammation is linked to asthma severity and control, our study shows that LCI detects residual airways disease independent of inflammation as our patients had largely normal FeNO that did not correlate with LCI.

In children with asthma there is evidence of progressive airway changes into adulthood despite good control, with reduced lung function and increased AHR in those with the longest duration of asthma. Saglani et al have shown that early structural changes to the airway wall are present in preschool children (3-5 years) with wheeze. ${ }^{5}$ As airway remodelling begins at an early stage, improvements in the treatment of children of preschool age may result in less airway remodelling, better lung growth and, ultimately, better lung function in adulthood.

In this study, LCI was able to detect residual airways disease following salbutamol administration in children with well controlled asthma. MBW may be a useful tool to track the progression of early airway structural disease that is not currently detected by spirometry.

Acknowledgements: The authors thank the volunteers for taking part in the study, Ann McMurray and the asthma clinic team for help with recruitment, and Per Gustafsson and Eddie Bergsten for help with the initial set-up of equipment and development of the software for analysis of multiple breath washout data.

Funding: This study was funded by the Sick Kids Respiratory Endowment Fund.

Competing interests: None.

Ethics approval: Approval for the study was given by the Lothian Research Ethics Committee.

\section{REFERENCES}

1. Kiley J, Smith R, Noel P. Asthma phenotypes. Curr Opin Pulm Med 2007;13:19-23.

2. Warke TJ, Fitch PS, Brown V, et al. Outgrown asthma does not mean no airways inflammation. Eur Respir J 2002;19:284-7.

3. van den Toorn LM, Overbeek SE, de Jongste JC, et al. Airway inflammation is present during clinical remission of atopic asthma. Am J Respir Crit Care Med 2001;164:2107-13.

4. Edwards CA, Osman LM, Godden DJ, et al. Wheezy bronchitis in childhood: a distinct clinical entity with lifelong significance? Chest 2003;124:18-24.

5. Saglani S, Malmstrom K, Pelkonen AS, et al. Airway remodeling and inflammation in symptomatic infants with reversible airflow obstruction. Am J Respir Crit Care Med 2005;171:722-7.

6. Saglani S, Payne DN, Zhu J, et al. Early detection of airway wall remodeling and eosinophilic inflammation in preschool wheezers. Am J Respir Crit Care Med 2007;176:858-64.
7. Macklem PT. The physiology of small airways. Am J Respir Crit Care Med 1998;157(5 Pt 2):S181-3.

8. Gustafsson PM. Inert gas washout in preschool children. Paediatr Respir Rev 2005;6:239-45.

9. Gustafsson PM, de Jong PA, Tiddens HA, et al. Multiple-breath inert gas washout and spirometry versus structural lung disease in cystic fibrosis. Thorax 2008;63:12934.

10. Stocks J. Lung function testing in infants. Pediatr Pulmonol Supp/ 1999;18:14-20.

11. Bush A. Asthma research: the real action is in children. Paediatr Respir Rev 2005;6:101-10.

12. Horsley AR, Gustafsson PM, Macleod KA, et al. Lung clearance index is a sensitive repeatable and practical measure of airways disease in adults with cystic fibrosis. Thorax 2008;63:135-40.

13. Aurora P, Kozlowska W, Stocks J. Gas mixing efficiency from birth to adulthood measured by multiple-breath washout. Respir Physiol Neurobiol 2005;148:125-39.

14. Aurora P, Gustafsson P, Bush A, et al. Multiple breath inert gas washout as a measure of ventilation distribution in children with cystic fibrosis. Thorax 2004;59:1068-73.

15. Aurora P, Bush A, Gustafsson P, et al. Multiple-breath washout as a marker of lung disease in preschool children with cystic fibrosis. Am J Respir Crit Care Med 2005; 171:249-56.

16. Gustafsson PM. Peripheral airway involvement in CF and asthma compared by inert gas washout. Pediatr Pulmonol 2007:42:168-76.

17. Beydon N, Davis SD, Lombardi E, et al. An Official American Thoracic Society/ European Respiratory Society Statement: pulmonary function testing in preschool children. Am J Respir Crit Care Med 2007;175:1304-45.

18. Downie SR, Salome CM, Verbanck S, et al. Ventilation heterogeneity is a major determinant of airway hyperresponsiveness in asthma, independent of airway inflammation. Thorax 2007;62:684-9.

19. Verbanck S, Schuermans D, Paiva M, et al. Nonreversible conductive airway ventilation heterogeneity in mild asthma. J App/ Physiol 2003;94:1380-6.

20. Verbanck S, Schuermans D, Noppen M, et al. Evidence of acinar airway involvement in asthma. Am J Respir Crit Care Med 1999;159:1545-50.

21. Gustafsson PM, Kallman S, Ljungberg $H$, et al. Method for assessment of volume of trapped gas in infants during multiple-breath inert gas washout. Pediatr Pulmonol 2003;35:42-9.

22. Verbanck S, Schuermans D, Van Muylem A, et al. Ventilation distribution during histamine provocation. J Appl Physiol 1997;83:1907-16.

23. Anon. Guidelines for the measurement of respiratory function. Recommendations of the British Thoracic Society and the Association of Respiratory Technicians and Physiologists. Respir Med 1994;88:165-94.

24. Miller MR, Hankinson J, Brusasco V, et al. Standardisation of spirometry. Eur Respir J 2005;26:319-38.

25. Rosenthal M, Bain SH, Cramer D, et al. Lung function in white children aged 4 to 19 years: I-Spirometry. Thorax 1993;48:794-802.

26. ATS/ERS. Recommendations for standardized procedures for the online and offline measurement of exhaled lower respiratory nitric oxide and nasal nitric oxide, 2005. Am J Respir Crit Care Med 2005;171:912-30.

27. BTS/SIGN. British guideline on the management of asthma. Thorax 2003;58/Suppl 1):i1-94.

28. Green RH, Brightling CE, McKenna S, et al. Asthma exacerbations and sputum eosinophil counts: a randomised controlled trial. Lancet 2002;360:1715-21.

29. Smith AD, Cowan JO, Brassett KP, et al. Use of exhaled nitric oxide measurements to guide treatment in chronic asthma. N Engl J Med 2005;352:2163-73.

30. Pijnenburg MW, Bakker EM, Hop WC, et al. Titrating steroids on exhaled nitric oxide in children with asthma: a randomized controlled trial. Am J Respir Crit Care Med 2005;172:831-6. 